\title{
DOSSIER
}

De Prácticas y discursos Universidad Nacional del Nordeste Centro de Estudios Sociales I Año 8, Número 11, 2019, Marzo I ISSN 2250-6942

\section{SALUD MENTAL Y GUBERNAMENTALIDAD: REFLEXIONES EN TORNO A LA LOCURA EN CHILE}

\section{MENTAL HEALIH AND GOVERNANCE: REFLECTIONS ON MADNESS IN CHILE}

\section{Alejandro Castro 2}

RESUMEN Nos enfrentamos a una época en la que los trastornos psiquiátricos proliferan en el mundo, y en la que la psiquiatría, a través de la salud mental, ha necesitado reconfigurarse para lograr su intervención a escala global. Para realizar ello, se ha generado una serie de medidas tanto a nivel sanitario como político. Una de ellas es el modelo de salud mental comunitaria, que en Chile se ha convertido en el modelo hegemónico de intervención en este campo. De ese modo, este artículo analiza cómo la salud mental se ha convertido en un modo de gobierno, gestionando el sufrimiento psíquico de las poblaciones a través de una serie de dispositivos de seguridad en torno a la locura. Se examina de manera breve cómo se instala en la retina social este modelo de gobierno y la administración del sufrimiento psíquico que padecen los sujetos locos y no locos. Para ello, se trae a la discusión los elementos principales del enfoque de la gubernamentalidad y la racionalidad neoliberal que de algún modo da forma al fenómeno de la salud mental y su despliegue actual en las políticas gubernamentales chilenas.

Palabras clave: salud mental, gubernamentalidad, biopolítica, locura.

1 Artículo recibido 15 de octubre de 2018. Aceptado 21 de abril de 2019 .

2 Licenciado en Trabajo Social, Magister en Estudios Sociales y Políticos Latinoamericanos y Doctorando en Ciencias Sociales. Universidad Alberto Hurtado Chile. Correo electrónico: alejandrocastroharrison@gmail.com

\section{INTRODUCCIÓN}

Preocuparse de la salud mental en la actualidad es pensar en sí mismo los modos de vida de la sociedad contemporánea. Al ser una parte integral de la salud y el bienestar humano, la salud mental se encuentra inmersa al interior de una serie de fenómenos sociales que la envuelven, transforman y determinan, con consecuencias directas en las emociones y cuerpos de los individuos. Los individuos y sus interacciones, la pobreza, el orden social y las intervenciones del Estado están de algún u otro modo imbricados en el devenir de la salud en general y, especialmente, en la salud mental.

En la actualidad, la salud mental está íntimamente ligada a lo comunitario, especialmente en Chile, donde se ha definido como el principal modelo asistencial de intervención sanitaria relacionada con las enfermedades mentales (MINSAL, 2014; Minoletti, 2010; Desviat, 2012). Estas directrices han sido definidas como una estrategia de gobierno que ha permitido colocar sistemáticamente una racionalidad teórica, organizativa, asistencial en la intervención relacionada con las enfermedades mentales. Especialmente en Chile se adopta esta medida ya que había razones éticas relacionadas con los DDHH en torno a la cuestión del manicomio y, por otro lado, razones técnicas relacionadas con la enfermedad mental y una profundización de una atención terapéutica y social. De acuerdo a Desviat (2010), los objetivos comunes eran la transformación o cierre de los hospitales psiquiátricos y la creación de recursos en la comunidad; la integración de la atención psiquiátrica en los sistemas de salud general; la modificación de la legislación que favoreciera los derechos de las personas con alguna discapacidad intelectual (que solo en algunos paises se ha logrado realizar) y promover el cambio en la representación social estigmatizante que tienen las enfermedades psiquiátricas.

En ese sentido, la actual salud mental es un guía que integra una clínica que rompe con las dicotomías individuo-sociedad, biológico-psicológico, basado en un paradigma científico centrado en el cuidado y la ciudadanía. Algunos, como Goldberg (1990), la definieron como un cruce sustantivo entre clínica y política, y otros, como Desviat (2010), dirán que no es más que la represen- 
De Prácticas y discursos

Universidad Nacional del Nordeste

Centro de Estudios Sociales

Año 8, Número 11, 2019, Marzo

ISSN 2250-6942

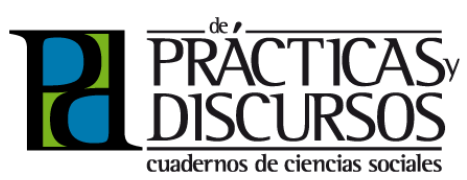

ABSTRACT We are facing a time when psychiatric disorders are proliferating in the world, and where psychiatry, through mental health, has needed to reconfigure itself to achieve its intervention on a global scale. To do this, a series of measures have been generated both at the health and political levels. One of them is the community mental health model, which in Chile has become the hegemonic model of intervention in this field. In this way, this article analyzes how mental health has become a mode of government, managing the mental suffering of populations through a series of security devices around madness. It is briefly examined how this model of government and the administration of the psychic suffering suffered by the mad and not mad subjects are installed in the social retina. To this end, the main elements of the approach to governmentality and neoliberal rationality that in some way shapes the phenomenon of mental health and its current deployment in Chilean government policies are brought into the discussion.

Keywords: mental health, governmentality, biopolitics, madness. tación de un cambio en la propia psiquiatría y, por tanto, implica una nueva actitud en el quehacer cotidiano de las profesiones sanitarias y, por supuesto, una planificación y ordenación de los sistemas públicos de salud mental.

En este contexto, y a fin de lograr un examen exploratorio acerca del funcionamiento de la salud mental en Chile, particularmente en su raciocinio de gobierno, el enfoque de gubernamentalidad nos permitirá introducirnos en una lectura estratégica para pensar cómo las ciencias psiquiátricas han instalado un modo de ser a través de ciertas instituciones, procedimientos y reflexiones que tienen por objetivo el control de la población con enfermedad mental, como también las que no sobrellevan una. Para ello, el enfoque de la gubernamentalidad -extraida desde los textos foucaultianos- nos permitirá un desmontaje de esa racionalidad del gobierno de la locura, especialmente en tiempos neoliberales. Ya en la Arqueología del Saber (2015), Foucault montaba una crítica a la medicina psiquiátrica a partir del análisis de las formas de representación de la locura como producto sociocultural, así también su concienzudo análisis sobre los dispositivos psiquiátricos y sus formas de violencia que están inmersas en una serie de estrategias normadas por discursos y saberes que terminaron gestionando un régimen de "verdad" (Foucault, 2008).

En la actualidad, la salud mental se organiza como un eje cardinal de políticas en salud pública a nivel mundial y no es sino la OMS (2013: 6) quien lo afirma al decir que: "no hay salud sin salud mental". De ese modo, hoy toma una importancia trascendental volver al examen cuidadoso de lo que significa este fenómeno que se inscribe más allá de la propia medicina.

Es por ello que un acercamiento desde la perspectiva de la gubernamentalidad permitirá reflexionar sobre los discursos de bienestar que se asocian a la salud mental, pero, a su vez, conseguiremos mirar cómo se ha construido un discurso específico sobre la locura. El propósito de este artículo es justamente develar algunas implicancias biopolíticas y gubernamentales que la salud mental tiene hoy en día, y cómo se construye y dispone en nuestras sociedades, especialmente en la chilena. En ese sentido, la perspectiva gubernamental nos introducirá a un análisis del estado actual de la gestión de la salud mental chilena, permitiendo observar la consolidación hegemónica del discurso psiquiátrico y la reducción de la complejidad del sufrimiento humano a un modelo simplista de síntomas, diagnósticos y tratamiento. Este hecho se podrá advertir 


\begin{abstract}
${ }^{3}$ Según la OMS, la depresión afectaría a aproximadamente 350 millones de personas en el mundo, siendo la primera causa de discapacidad según esta entidad. Como consecuencia de ello, cada año se suicidarían alrededor de 800000 mil personas, siendo la segunda causa de muerte en el grupo etario entre 15 a 29 años. Disponible en: http://www.who.int/mediacentre/factsheets/fs369/ es/, consultado el 10 de octubre de 2018.
\end{abstract}

a partir de cómo se instala el modelo comunitario en la política de salud mental chilena, determinando el campo de intervención, y como consecuencia, una consolidación de prácticas individualizadoras y biologicistas que cosifican el sufrimiento psíquico.

\section{LOCURA, SALUD MENTAL Y GLOBALIZACIÓN}

La salud mental se ha vuelto un elemento importante a nivel global, sobre todo cuando se viven tiempos de alta desigualdad social provocada -entre otras- por las actuales crisis financieras, ambientales, sociales y políticas, teniendo consecuencias directas en los cuerpos y las emociones de los individuos. Los altos índices de sobrecarga laboral, por ejemplo, han hecho emerger nuevos fenómenos en salud, tales como el sindrome de desgaste ocupacional o más conocido como el burnout. También, las tasas de depresión han aumentado exponencialmente en los últimos diez años, logrando que esta sea una de las enfermedades más diagnosticadas en el mundo ${ }^{3}$. Junto con estas crisis, los incrementos de los efectos de las masivas migraciones y el recrudecimiento del racismo en las sociedades actuales afectan directamente la vida de las personas y su salud mental. Como consecuencia de ello, nos encontramos con niños expuestos al maltrato y abandono, con discriminaciones y violaciones a los derechos humanos, entre otros fenómenos.

En este contexto, la salud mental se ha vuelto un problema público, pues relaciona la enfermedad mental con problemas sociales tales como la marginación, el empobrecimiento, la violencia y el maltrato doméstico, el exceso de trabajo y el estrés. Aun cuando este trabajo no pretende abordar estos problemas estructurales, es importante mencionar cómo las sociedades perciben sus experiencias humanas, a tal punto que para explicarlas deben recurrir a comprensiones médicas para justificar sus acciones. Byung-Chul Han (2016: 17) Ilamará al siglo XXI como una época neuronal, donde todas enfermedades emblemáticas tales como la depresión, los trastornos de personalidad, etc., son, por definición, enfermedades representativas de esta época:

Lo que provoca la depresión por agotamiento no es el imperativo de pertenecer solo a sí mismo, sino la presión por el rendimiento. Visto así, el síndrome de desgaste ocupacional no pone de manifiesto un sí mismo agotado, sino más bien un alma agotada. [...] En realidad, lo que enferma no es el exceso de responsabili- 
De Prácticas y discursos

Universidad Nacional del Nordeste

Centro de Estudios Sociales

Año 8, Número 11, 2019, Marzo

ISSN 2250-6942

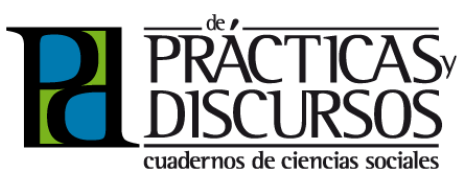

dad e iniciativa, sino el imperativo del rendimiento como nuevo mandato de la sociedad del trabajo tardomoderna. (Byung-Chul Han, 2016: 29)

El diagnóstico que hace el autor nos coloca en un escenario complejo que relaciona un modo de vida en tiempos neoliberales y las enfermedades mentales. La obligación que se tiene como trabajador en el hecho de maximizar su rendimiento, ha tenido como resultado un desequilibrio emocional que conlleva al sufrimiento psíquico de los individuos. De algún modo, la depresión sería consecuencia de ello. Desde el punto de vista sociológico, específicamente marxiano, el exceso de trabajo y rendimiento se agudiza y convierte el sentido humano del trabajo en una autoexplotación eficaz, que estaría acompañada de un sentimiento de libertad (Han, 2016: 32). En otras palabras, el explotador es al mismo tiempo explotado $y$, de ese modo, las enfermedades psíquicas son la consecuencia directa de esa sociedad del rendimiento, a la que Han hace referencia, constituyéndose como manifestaciones patológicas de esa libertad paradójica. Sin embargo, las ciencias de la salud, especialmente la medicina psiquiátrica, tendrán otras explicaciones que más bien están vinculadas a razones biológicas y sanitarias. La medicina en general reconoce que el contexto mundial y las grandes crisis financieras y sociales tienen un gran impacto en las personas. Estas generan desastres sociales que favorecen las patologias psíquicas, sin embargo, difieren sustancialmente en las explicaciones sobre su origen en los individuos (que para gran parte de la comunidad médica estarán asociadas a los desequilibrios neuronales). A pesar de lo anterior, se muestran de acuerdo en que los efectos que tienen en los individuos han de tener como consecuencia la exclusión de las personas que padecen trastornos mentales (en distintas medidas de acuerdo a la cronicidad de ellas). La OMS en este sentido nos dirá que:

Con frecuencia, los trastornos mentales hunden a las personas y a las familias en la pobreza. La carencia de hogar y la encarcelación impropia son mucho más frecuentes entre las personas con trastorno mental que en la población en general, y exacerban su marginación y vulnerabilidad. Debido a la estigmatización y la discriminación, las personas con trastornos mentales sufren violaciones de los derechos humanos, y a muchos se les niegan derechos económicos, 
sociales y culturales, y se les imponen restricciones en el trabajo y a la educación, así como a los derechos reproductivos [...] Pueden sufrir también condiciones de vida inhumanas $y$ poco higiénicas, maltratos físicos y abusos sexuales, la falta de atención y prácticas terapéuticas nocivas y degradantes en los centros sanitarios. A menudo se les niegan derechos civiles y políticos, tales como el derecho a contraer matrimonio y fundar una familia, la libertad personal, el derecho de voto y de participación plena y efectiva en la vida pública, y el derecho a ejercer su capacidad jurídica en otros aspectos que les afecten, en particular el tratamiento y la atención. Así, las personas con trastornos mentales suelen vivir situaciones de vulnerabilidad y pueden verse excluidas y marginadas de la sociedad [...]. (OMS, 2013:8)

De esta manera, la salud mental se vuelve un problema global de plena vigencia y estaría asociada a temas que van desde el estrés, el cansancio, hasta cuestiones más complejas como los trastornos psiquiátricos mayores (la esquizofrenia, la depresión severa y el trastorno afectivo bipolar). Más allá de cualquier explicación, las dificultades vinculadas a la salud mental -antiguamente llamada locura- tienen efectos negativos en los sujetos y sus experiencias cotidianas, en donde el sufrimiento psíquico se ve administrado y gestionado por unos dispositivos tecnológicos que la psiquiatría ha puesto en la escena sanitaria.

\section{UNA BREVE HISTORIA DE LA LOCURA}

Para dar cuenta de este conjunto de problemas, debemos retroceder un par de decenios y entender cómo la cuestión del loco y la psiquiatria emergen en las sociedades actuales. La locura se ha constituido históricamente como un fenómeno que nos ha acompañado socialmente a través del tiempo y que se ha ido perfilando como una forma de entender al mundo bajo la sin-razón. Sin embargo, si queremos definirla, la verdadera pregunta estaría centrada más bien ¿en qué consiste estar simplemente loco? Ya sea la demencia, la enajenación, la esquizofrenia, el suicidio, el idiotismo o simplemente la enfermedad mental, la etiquetación asociada a la locura significará una vinculación negativa del sufrimiento psíquico.

Desde el castigo divino, presente en los mitos originarios, tanto en la Grecia antigua como en la cultura judeo-cristiana, la locura 
De Prácticas y discursos

Universidad Nacional del Nordeste

Centro de Estudios Sociales

Año 8, Número 11, 2019, Marzo

ISSN 2250-6942

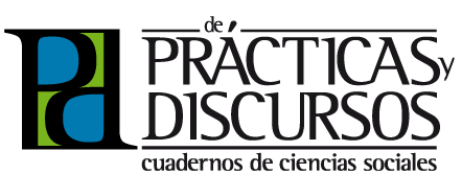

no es más que la desobediencia contra los dioses. No obstante, la Modernidad trajo consigo el bastión de la racionalidad y lo científico, en donde la locura queda desprovista de ese castigo divino, debiendo transformar esa explicación en una elucidación científica en donde la definición más importante la encontraremos en la enfermedad mental.

La locura como una patología mental se abre sobre un universo moral en donde la enfermedad es una falta y, como tal, es una forma desviada de percibir el mundo. Tal explicación se alejará inmediatamente de la experiencia del sufrimiento psíquico de las personas, y esta nueva lógica se fundará en lo que se denominó tratamiento moral, del que Philippe Pinel indicaba que era la manera más adecuada para tratar dichas desviaciones (Pinel, 1988). Para este médico, las cárceles no son un lugar digno para los locos, gestionando una nueva forma racional de intervenir a través de un dispositivo institucional que hasta el día conocemos como el Manicomio. Para Pinel, liberar al loco de la cárcel y reinstalarlo en un espacio asilar de orden médico significará el primer lugar de trato digno a quienes padecen una enfermedad mental. Dicho tratamiento estaba basado en el ejercicio físico y manual (lo terapéutico) para combatir lo que se consideraba el principio de la enfermedad mental: el ocio. El tratamiento moral -como bien indica el término- buscaba un mayor desarrollo moral del loco y mejorar, a través del disciplinamiento, las conductas de los enfermos mentales. En esa misma línea, Benjamín Rush en EE.UU. homologará esta experiencia creando una red asilar a lo largo de Norteamérica. Esta fue la primera red institucional para enfermos mentales en América (Gabay, 1999).

El siglo XVIII da comienzo a una organización racional de la enfermedad mental y donde "el espacio de clasificación se abre sin problemas al análisis de la locura, y la locura, a su vez, encuentra inmediatamente su lugar alli [...]" (Foucault, 2007: 273). Emerge una nueva transformación de la enfermedad mental: la clasificación psiquiátrica, que condujo a estudiar la locura de manera distinta, como una enfermedad mental basada en sintomas.

La locura, entendida como lo irracional y lo desviado, se cosificará en la institución moral de la psiquiatría científica a través de una racionalidad positivista que la concebirá como una enfermedad y no como una experiencia, relevando el sintoma por sobre la persona. Según Thomas Szasz (1970), cuando hablamos de enfermedad mental, estamos en un lugar ideológico, pues lo 
que existe es una maniobra política en donde la psiquiatría aprehende el sufrimiento psíquico de los individuos transformándola en una enfermedad. Según Reed y Geekie (2012), esta controversia aún persiste en la psiquiatría, y la discusión sobre enfermedad mental, discapacidad y locura permanece como un lugar de apropiación ideológica por parte del discurso psiquiátrico. A comienzos del siglo XX se produce una nueva habilitación para la noción de locura, hasta ese momento entendida como enfermedad mental. Los padres de la psiquiatría moderna, así llamados por esta disciplina, Emil Kraepelin y Eugen Bleuler, llevarán un paso más allá a la psiquiatría en términos científicos: crearian un principio clasificatorio de sintomas, dando nacimiento a lo que hasta hoy se denomina demencia temprana (Kreapelin, 1913) o esquizofrenia (Bleuler, 1924). Esta operación fundante emergió como forma de ordenamiento disciplinar, apropiándose de la enfermedad mental, los sintomas de un individuo y las categorizaciones definidas (ahora científicamente) que, bajo estudios de corte empirista, constataban el nacimiento de una nueva enfermedad a partir de la acumulación de sintomas.

Tales sintomas estarían basados en las conductas desviadas de aquella época. Kraepelin definía que la demencia temprana estaba caracterizada porque

se comportan de manera libre y espontánea, se ríen en las situaciones serias, actúan con groserías e impertinencia hacía sus superiores, les desafían, pierden la compostura y la dignidad personal; llevan ropas sucias y van desaseados y descuidados, entran en la iglesia con el cigarro encendido en la boca. (Kraepelin, 1913: 23)

En esa misma línea, Bleuler caracteriza las conductas esquizofrénicas porque "a menudo se observan perversiones como la homosexualidad o anomalias parecidas en la conducta general y en el atuendo del paciente" (Bleuler, 1924: 188).

Como advertimos, las enfermedades mentales estarian directamente asociadas con las conductas anormales que determinan cada sociedad, a lo que se suma la tendencia de que un grupo de individuos expertos establezca que tales desviaciones sean consideradas como enfermedad mental. De ese modo, emergen los diagnósticos psiquiátricos que marcan la diferenciación entre los desviados y los normales. 
De Prácticas y discursos

Universidad Nacional del Nordeste

Centro de Estudios Sociales

Año 8, Número 11, 2019, Marzo

ISSN 2250-6942

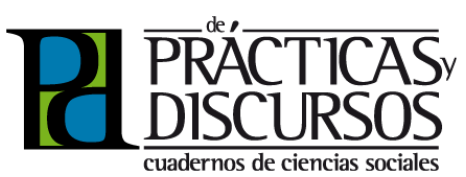

\section{DE LA ANTIPSIQUAITRÍA A LA RED DE SALUD MENTAL}

A partir de los años sesenta, el psiquiatra italiano Franco Basaglia inició un proceso revolucionario en torno a la idea de la psiquiatría, básicamente al darse cuenta (como director de un manicomio) que los internos o "locos" recibian un trato carcelario. Basaglia pronto comenzó a repensar la institución total -en el sentido goffmaniano- democratizando los espacios de la psiquiatría a partir de prácticas más humanas en torno al loco. La experiencia de Gorizia (Basaglia, 1972) significó una crítica al modelo hegemónico de la psiquiatría de ese tiempo y permitió generar un movimiento político antipsiquiátrico que prontamente se ramificaría por distintos lugares de Europa y América. Basaglia pensaba que los métodos ejercidos por la medicina psiquiátrica consistían en prácticas tortuosas para los pacientes diagnosticados con alguna enfermedad mental, intentando humanizar los tratamientos psiquiátricos como el trato hacia al loco. Por otro lado, decía que el pensamiento racional de la Modernidad habría subyugado a la irracionalidad y que, por medio de instituciones como la cárcel y el manicomio, esta podía ser dominada. Basaglia dice que:

Es importante observar que los manicomios nacieron en un momento en el que el mundo estaba cambiando y nacía un nuevo humanismo [...]. Una sociedad para ser civil, debe ser racional. Es por eso que de ese momento todo aquello que es irracional debe ser controlado por la razón. Es así como nace la institución racional del manicomio, que encierra la irracionalidad. (Basaglia, 2008: 58)

Es en este escenario donde se da comienzo al movimiento antipsiquiátrico, con un propósito determinado, que es la desmantelación del orden psiquiátrico hegemónico de esa época y que se representaba principalmente a través del encierro forzoso. Este pensamiento antipsiquiátrico fue acuñado por David Cooper en su famosa obra llamada Psiquiatría y Antipsiquiatría, de 1967. Otros autores que habrian influenciado sustancialmente este movimiento son el psiquiatra escocés Ronald Laing, con su obra El yo dividido: Un estudio sobre la Salud y Enfermedad (1975), además del nombrado Franco Basaglia.

De ese modo, los movimientos sociales relacionados con la antipsiquiatría habrian terminado en un proceso reivindicativo de 
los derechos de quienes padecian un sufrimiento psíquico, generando lo que se denominó como la desinstitucionalización de la locura. Esto no es más que la liberación del encierro, el manicomio y la deshumanización de este sujeto. Ello se plasmó con la Ilamada Ley Basaglia en 1978, en Italia. Esta regulación legal permitió una democratización de la psiquiatría y por primera vez empuja al Estado a hacerse cargo de las condiciones humanas de las personas con problemas de salud mental, con un fuerte énfasis basado en los derechos humanos.

Sin embargo, este movimiento habría sido obnubilado por los grandes cambios económicos en Europa Occidental y EE.UU., a propósito de la llegada de la economía global, sufriendo variadas modificaciones entre los años setenta y noventa. La noción de anti-psiquiatria fue perdiendo uso (básicamente su contenido revolucionario), redefiniéndose con una noción "comunitaria". Tanto en Inglaterra como en EE.UU. se rediseñó la noción de antipsiquiatría y se vinculó su significado con la noción de salud mental basada en la comunidad, pero sin eliminar el encierro. Si bien este ya no era el manicomio clásico (que sí se mantuvo, al menos en EE.UU.), la psiquiatría se desplazó hacia el interior del Hospital General. Según críticos como el profesor Carlos Pérez Soto (2012), esto se debió a una respuesta economicista, esencialmente porque los manicomios efectivamente generaban un gasto público excesivo, en un contexto económico neoliberal. Por tanto, el cálculo indicaba que era más rentable para un Estado tener al loco fuera de este tipo de instituciones, vigilado y controlado, que encerrado en instituciones que generaban altos gastos financieros. Además, esto iba a favor de los nuevos tiempos, donde la libertad se constituía como un bastión del neoliberalismo y donde las sociedades democráticas comenzaban a proliferar, especialmente en Latinoamérica.

Chile no se vio ausente en esta transformación racional de la psiquiatría. El tratamiento de la locura se fundó históricamente en el modelo asilar de la Casa de Orates, creada en 1852, y que subsistió hasta 1928. Posteriormente se denominó "Manicomio Nacional", y de ese modo proliferaron otras instituciones totales como el manicomio El Peral en la zona sur de Santiago, dependiente del Manicomio Nacional. Posteriormente, en 1955, la ex Casa de Orates pasa a llamarse Instituto Psiquiátrico hasta 1983, cuando cambia el nombre a Instituto Psiquiátrico Dr. José Horwitz Barak, hasta la fecha. En consecuencia, las políticas de la 
De Prácticas y discursos

Universidad Nacional del Nordeste

Centro de Estudios Sociales

Año 8, Número 11, 2019, Marzo

ISSN 2250-6942

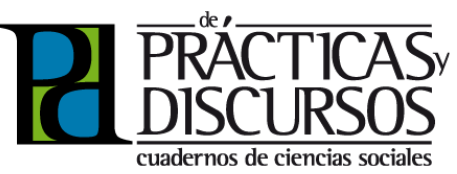

locura en nuestro país sostuvieron el modelo asilar como forma de tratamiento en continuidad con los modelos tradicionales de la psiquiatría clásica.

A pesar de lo anterior, en la parte final de los años sesenta e influenciado por el movimiento que buscaba la desinstitucionalización de la locura en los países europeos, el psiquiatra chileno Juan Marconi impulsó un modelo de salud mental comunitaria entre 1968 y 1973 desde la Facultad de Medicina de la Universidad de Chile. Tal empresa tuvo un énfasis que se centraba en los derechos ciudadanos de los pacientes con problemas de salud mental. La experiencia desarrollada por Marconi, denominada como el "programa intracomunitario", entendía a la salud mental como un modelo complementario al hospital general donde se desarrollaba la intervención en salud mental. Marconi proponia una serie de reformulaciones relacionadas con la psiquiatría y el hospital general, en donde estos dos se articularán de manera sinérgica, como una "red de instituciones dentro y fuera del hospital general que comprende la atención primaria [...], postas rurales, atención secundaria [...], urgencia psiquiátrica, psiquiatría enlace, hospitalización diurna, nocturna y completa, rehabilitación en hogares y talleres protegidos" (Pemjean, 2006: 73). Esta red de atención, que es la protoforma del modelo de salud mental actual en Chile, intentaba extender una red de atención psiquiátrica y usar los recursos propios de las comunidades locales:

En efecto, luego de reconocer, validar y respetar la influencia de los familiares, los grupos comunitarios próximos, las instituciones propias de la comunidad, confía en entregarles un adiestramiento tecnológico "mínimo suficiente". Surgen así, líderes de salud mental, grupos de ex pacientes (alcoholismo, trastornos emocionales), grupos organizados de familiares de pacientes y otros, quienes asumen roles pre-especificados en prevención, co-terapias y fundamentalmente en rehabilitación, dentro de un marco de trabajo en equipo [...]. (Pemjean, 2006: 73)

El modelo intracomunitario marconiano vinculaba el mundo psiquiátrico y hospitalario con la comunidad, otorgando a estos últimos ciertas capacidades para reconocer procesos de prevención y protección en relación con las enfermedades mentales. Sin embargo, la dictadura militar descabezó esta iniciativa por contener cierta ideología peligrosa para el régi- 

y la articulación de base, peligrosa para los momentos de intervención militar en el pais, fueron desmembrados, retornando a las lógicas manicomiales durante todo el proceso dictatorial, cerrando cualquier posibilidad a una apertura a una salud mental comunitaria.

La vuelta a la democracia trae consigo una redimida psiquiatría comunitaria a partir de los años noventa. Ya con la práctica inglesa, española y norteamericana como experiencias concretas, se instala la idea de la locura fuera del manicomio, sin dejar de entender a este fenómeno como un proceso medicalizador. Según los psiquiatras impulsores de este modelo:

En la década de los años noventa, con la recuperación de la democracia, se incorporaron a los programas de atención un conjunto de acciones destinadas a garantizar los derechos humanos de las personas afectadas por trastornos mentales. Fue la época cuando el país dio los primeros pasos para adoptar en el ámbito de la salud mental un nuevo modelo de atención acorde con las recomendaciones de los organismos de salud y con los progresos en otros países en esta materia. (Minoletti et al., 2010: 339).

El modelo comunitario de la psiquiatría posdictadura se habría coronado con la primera Estrategia Nacional de Salud Mental (MINSAL, 1993) en 1993, que a través de una intervención de carácter público mejora el acceso a la salud mental. Sin embargo, no fue sino hasta el 2000, tras una larga discusión entre las autoridades y la sociedad civil, cuando se logra desarrollar e implementar el Plan Nacional de Salud Mental ${ }^{4}$ (MINSAL, 2000), desinstitucionalizando los pacientes de los manicomios (pacientes psiquiátricos que estaban destinados de por vida en las instituciones totales), siendo reubicados en nuevos dispositivos de carácter comunitario llamados Hogares Protegidos (MINSAL, 2000). Otros retornaron con sus familias en sus propias comunidades. En esa misma línea se propone una cierta división territorial para el control de las personas con problemas de salud mental, a través de los hospitales generales, basada en una atención ambulatoria y territorializada, comenzando a desarrollarse una red pública de la salud mental que tiene por objetivo crear un sistema de atención a nivel país en este ámbito. 
De Prácticas y discursos

Universidad Nacional del Nordeste

Centro de Estudios Sociales

Año 8, Número 11, 2019, Marzo

ISSN 2250-6942

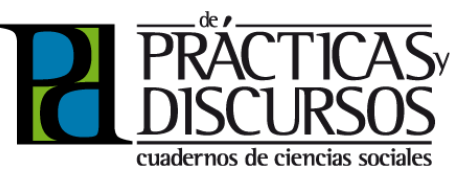

\section{LA SALUD MENTAL CHILENA COMO UN MODO DE GOBIERNO}

La instalación del modelo de salud mental comunitaria que se da a partir del PNSM se comienza a implementar como política de Estado desde el 2000. Este viene a resaltar un cambio paradigmático en la forma de intervenir la salud mental chilena, proponiendo una división territorial de la población que padece una enfermedad mental. La centralidad de la salud mental, ahora detentada por los hospitales generales, busca articularse con las redes territoriales de atención psiquiátrica, modificando las formas de intervenir la locura a nivel pais. De ese modo comenzó a proliferar una red de atención a nivel comunitario, a través de la creación de un entramado de dispositivos relacionados con la salud mental y la despotencialización de los hospitales psiquiátricos chilenos. A ello se agregó una política gubernamental de acceso al tratamiento de enfermedades psiquiátricas de mayor complejidad como son la esquizofrenia, el trastorno afectivo bipolar y la depresión (entre 2008 y 2013). Las Garantías Explicitas de Salud (GES) fueron la manera en que se denominó este mecanismo de intervención gubernamental que proporcionó un financiamiento público a la atención psiquiátrica, garantizando el acceso en todos los niveles de atención del país (primaria, secundaria y terciaria, tanto pública como privada).

Esta nueva red de salud mental, conformada por distintos dispositivos territoriales y hospitalarios, se dispersa a través de los servicios de salud y los municipios de todo el país. Ello tiene como consecuencia una nueva forma de concebir la locura, la enfermedad mental o como ahora se denominan, los trastornos psiquiátricos. Esta política comunitaria significó el aumento de profesiones ligadas a esta área, fomentando una nueva forma de intervención social, especialmente la llamada biopsicosocial. En ese sentido, ya no es la psiquiatría solamente la que interviene en los problemas asociados con la locura, sino que otras disciplinas como la terapia ocupacional, el trabajo social, la psicología, entre otras.

La psiquiatría, en general, ha incorporado elementos de la crítica al poder disciplinar, alineándose con "las nuevas mentalidades de gobierno" (Carrasco, 2014: 149). De ese modo, si pensamos la actualidad de la salud mental en un marco de análisis foucaultiano, podríamos visualizar algunas racionalidades que se juegan al interior de este modelo, y principalmente de la empresa de la locura en Chile. La intervención social propuesta por el mode- 
lo comunitario de salud mental puede ser comprendido desde una perspectiva de la gubernamentalidad, a partir de una serie de "tecnologías" que busca organizar el control desde el Estado hacia las subjetividades de las personas y grupos sociales. La emergencia de lo comunitario desde esta perspectiva aparece como un nuevo lenguaje político que moldea las estrategias relacionadas con la salud mental y su intervención en el campo de las enfermedades mentales y todo lo que lo rodea (Rose, 2007). La creación del manicomio es la expresión de una cierta racionalidad gubernamental para el control social de los locos, y en si mismas, además de ser prácticas médicas, eran también prácticas políticas. El objetivo del manicomio en ningún modo era sanar-discurso que se mantiene hasta el día de hoy-, sino mantener un orden social en donde el loco no forma parte de ella por su calidad individuo irracional. Por medio del encierro se podian perfeccionar aquellas "tecnologías del yo", a través de la "clasificación, la vigilancia y la supervisión de los registros de internos" (Carrasco, 2014: 129), creando un orden institucional desarrollado por el saber experto del psiquiatra. La figura de este y su "eficacia simbólica" -en clave de Levi-Strauss- permiten que la medicina y la psiquiatría se establezcan como una expresión del poder del panóptico, a través de tecnologías disciplinares aplicadas sobre los cuerpos del loco. Lo novedoso de ello en la actualidad es que funcionaría tanto en el campo de la institución total (Goffman, 1998) como en la psiquiatría comunitaria.

Por otro lado, el psiquiatra se erigirá como un agente moral de las sociedades contemporáneas, determinando, de acuerdo a su saber disciplinar, quién es el "psiquiátrico" o no, es decir, quién está loco. Este ejercicio de poder, que está localizado en lo disciplinar, traspasará la frontera de la institución total. En otras palabras, la psiquiatría comunitaria, que evidenció su descontento con los modelos asilares (y sus fechorias en contra de los derechos humanos al interior de estos), trasladó el "tratamiento" a la "comunidad", es decir, el loco debe someterse a un tratamiento ambulatorio o comunitario, ya no en el encierro, sino en la libertad. Algo similar es a lo que se refiere Rose cuando habla de gobierno a través de la comunidad (Rose, 2007: 122), esto "implica una variedad de estrategias para inventar e instrumentalizar estas dimensiones de lealtad entre los individuos y las comunidades al servicio de proyectos de regulación, reforma o movilización". En ese sentido, la psiquiatría comunitaria viene a ser ese proyecto de 
De Prácticas y discursos

Universidad Nacional del Nordeste

Centro de Estudios Sociales

Año 8, Número 11, 2019, Marzo

ISSN 2250-6942

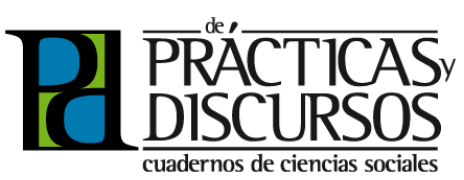

regulación que instrumentaliza las relaciones entre individuos y comunidades, y el PNSM, el mecanismo con el cual se echa a andar esa relación a través de los dispositivos de salud.

El PNSM del 2000 y el actual de 2017 (MINSAL, 2017) invocan una lógica democrática que lega en los Derechos Humanos y debe plasmarse en las intervenciones de salud mental comunitaria. Estas indican que "todo tratamiento debe ser voluntario, siempre y cuando este no ponga en peligro a sí mismo y/o terceros" (MINSAL, 1993). Sin embargo, los equipos de psiquiatría comunitaria han generado cierta institucionalidad al interior de la comunidad, a partir de la vigilancia y control en los dispositivos dependientes de los hospitales generales y de la comunidad (Centros diurnos, Hospitales de día, Unidades de Rehabilitación psicosocial, Atención Primaria, etc.), además de un vínculo con vecinos y familias cercanas al usuario de salud mental -su hogar- que favorecerán ese panóptico comunitario.

Intervenciones denominadas como la adherencia a tratamiento se transforman en mecanismos de seguridad, control y vigilancia, con el fin de asegurar una medicalización de la vida del loco. Ello se plasma en intervenciones como la fármacovigilancia que opera en los dispositivos de salud mental a manera de supervisar y controlar el uso (y consumo) de psicofármacos. Así es como también el rol de los profesionales de la salud mental se asemeja a una "policia" de la locura, vigilando y asegurando constantemente la adherencia al tratamiento del loco al interior de las comunidades donde estos residen. Cuando estos fallan, emergen mecanismos de coacción denominados internaciones forzosas que son herramientas legales que suprimen la libertad de las personas en pos de una estabilidad psicopatológica.

Por otro lado, la creación de los sistemas sanitarios de salud mental comunitaria se diseñó pensando en que el sujeto afectado a un problema de salud mental esté inserto en su medio social, es decir, su comunidad (casa, lugar de trabajo o un similar). Ello significará el involucramiento de una red de apoyo sostenida por las intervenciones biopsicosociales que deben generar las prácticas de los equipos territoriales, favoreciendo la estabilidad del usuario con las reglas establecidas del saber experto, la psiquiatría.

Para llevar a cabo tal trabajo, esta red se centralizó en equipos de salud interdisciplinarios al interior de una red comunitaria u hospital general, que está a cargo del control y vigilancia del tratamiento de las personas con problemas de salud mental. Es- 
tos equipos entrenan a las familias o redes de apoyo, además de interactuar con la atención primaria de salud, con el objetivo de poder desarrollar abordajes terapéuticos y farmacológicos en los "locos". En este sentido, lo fundamental para que un tratamiento se considere relativamente adecuado estará dado por el proceso de control farmacológico y terapéutico. En el primer caso, la toma de fármacos se constituye como el elemento central del tratamiento en salud mental, sin este no podría existir ninguna condición básica de relación entre los sujetos locos y la medicina psiquiátrica. Si este proceso se interrumpe, o se suspende, debe ser por una exclusiva orden del psiquiatra, pero si este es suspendido por las propias personas, los mecanismos coercitivos de los equipos de salud mental presionan en el engranaje de la red comunitaria y familiar para que esta se reactive. Sin embargo, si aún esto fallara, existirian los medios coercitivos como las hospitalizaciones involuntarias y forzosas, que obligarian al sujeto afectado a acceder al tratamiento psiquiátrico. En el caso de lo terapéutico, este opera como un dispositivo ambiental que se vincula afectivamente al malestar objetivo, pero desde una politica de experto en la intervención. De ese modo existe una validación terapéutica por parte de los pacientes en relación con el experto (el terapeuta), pudiendo realizarse "en la medida en que conductas, pensamientos, sentimientos $u$ otros aspectos de un sujeto se convierten en signos que deben ser descifrados y analizados, de manera de delimitar claramente aspectos de su subjetividad" (Carrasco, 2018: 172). Así, lo terapéutico se legitima tanto en un nivel de experto como también en la relación afectiva con quienes cursan el sufrimiento psíquico. Para Carrasco, tal manera de intervenir no es más que una mentalidad de gobierno que funciona a favor de la dominación de estos sujetos.

Tanto la medicalización como lo terapéutico en la salud mental comunitaria se organizan como un eje central en el tratamiento de los sujetos de la locura; no obstante, podríamos decir que el énfasis ya no se encuentra en la esquizofrenia misma, o en curar la depresión, sino más bien en mantener la vida saludable de estas personas. Precisamente ello caracteriza la medicalización indefinida a la que Foucault hace alusión en su obra (Foucault, 1996). Así las prácticas discursivas de la salud mental comunitaria se establecen apropiándose de otros campos sociales, por fuera de la enfermedad; por ende, se trata la salud y no las enfermedades mentales. Un ejemplo de ello son los programas de 
De Prácticas y discursos

Universidad Nacional del Nordeste

Centro de Estudios Sociales

Año 8, Número 11, 2019, Marzo

ISSN 2250-6942

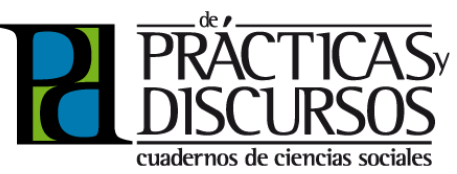

promoción y prevención en salud mental existentes en la red de atención primaria y secundaria de salud a lo largo de todo Chile, donde la cuestión de la población adquiere alcances inimaginables, generando efectos performativos en los discursos de las personas "normales".

En esta línea, la intervención gubernamental en atención primaria representa el mejor modelo de control biopolítico en las comunidades. El segundo reporte del sistema de salud mental chileno de 2014 muestra cómo se han generado campañas de sensibilización ciudadana en torno a los trastornos psiquiátricos, especialmente la depresión (MINSAL, 2014). Por otro lado, las actividades de promoción y prevención en salud mental han sido vinculadas paulatinamente a los sistemas educacionales (en coordinación con el Ministerio de Educación): "el 13,7\% de las escuelas primarias y secundarias del pais tienen al menos un profesional de la salud mental tiempo parcial o completo" (MINSAL, 2014: 87). La intervención en salud mental desde esta perspectiva ha alcanzado a otro tipo de institucionalidades como las de orden público, donde el 37,9\% de la policía chilena recibió capacitación sobre el abordaje en salud mental. Sin embargo, la mayor inversión en términos de recursos - para efectos de esta ejemplificación- ocurriria en la atención primaria. Desde la puesta en marcha del PNSM de 2002, la atención primaria ha concebido un aumento significativo en la atención de personas con problemas de salud mental. En ese sentido, el informe de OPS muestra que en la atención primaria chilena

aumentó el porcentaje de centros que cuentan con psicólogo, así como la cantidad de psicólogos y el promedio de horas que disponen por centro; también aumentó el porcentaje de médicos APS que interactúan con equipos de salud mental secundarios a través de consultorías; también aumentaron los porcentajes de centros que realizaron actividades de medicina alternativa y que trabajan con organizaciones de usuarios y familiares de salud mental. (MINSAL, 2014: 74).

Se trata entonces de una lógica gubernamental y de control biopolítico, sobre la base de un cambio estructural en la relación medicina-enfermedad o psiquiatría-locura. Se transporta la figura del psiquiatra y un equipo de salud mental especializado, hacia la comunidad donde el loco reside (y los posibles locos), 
convirtiéndose en autoridades sanitarias y sociales a nivel comunitario. Cambia la institucionalidad hospitalaria, colocando a los equipos clínicos de atención fuera de los hospitales generales en dispositivos comunitarios, modificando la relación tradicional médico-paciente de forma sustancial. De ese modo, emergen nuevas formas de control de datos, a través de una gestión comunitaria que involucra una red sanitaria de atención primaria y secundaria, además de social, al interior de la comunidad.

Podemos decir entonces que "la sociedad moderna en que vivimos es en 'Estados médicos abiertos' en los que la dimensión de la medicalización ya no tiene límites" (Foucault, 1996: 80), de esa forma se perpetúa un continuo control y vigilancia. Lo interesante de este punto es que ya no se produce al interior de la institución total, sino fuera de sus paredes, es decir, la comunidad, emergiendo otro tipo de institucionalidad mucho más panóptica y virtualizada, una especie de "post-institución total". Gilles Deleuze (1996) coincide con este análisis al decirnos que: "Estamos entrando en sociedades de control, que ya no funcionan mediante el encierro sino mediante un control continuo y comunicación instantánea" (1996: 243). El PNSM, a través de la psiquiatría comunitaria, se ha encargado de ir eliminando paulatinamente la figura del hospital como centro neurálgico de la salud mental, y a través de la medicalización indefinida y la intervención biopsicosocial se levantan como una nueva mentalidad de gobierno. Como consecuencia, se ha erigido una nueva manera de pensar el control y la vigilancia sobre el fenómeno de la locura, permitiendo un ensamblaje nuevo que despliega una intervención permanente en el territorio, a través de mecanismos articulados en redes que propician una vigilancia y control psiquiátrico sempiterno del enfermo mental.

\section{LA SALUD MENTAL COMO UN OBJETO DE GESTIÓN GUBERNAMENTAI}

El académico antipsiquiatra chileno Carlos Pérez Soto (2015: 19) dice:

Hay que considerar que un "enfermo mental" tranquilizado de manera profunda, en su propia casa, de algún modo oculto y apartado de cualquier forma de relación social, no solo representa un "éxito" desde el punto de vista del orden público, sino también una importante reducción de costo de los servicios de salud que los Estados se sentían obligados a dedicarle. 
De Prácticas y discursos

Universidad Nacional del Nordeste

Centro de Estudios Sociales

Año 8, Número 11, 2019, Marzo

ISSN 2250-6942

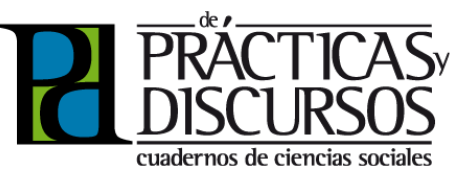

En 2018, al tiempo que se publica el nuevo PNSM, emerge un modelo de gestión como guía de funcionamiento de las redes de salud mental chilenas (MINSAL, 2018). Este documento es una carta de navegación de la gestión en salud mental, fundamentada principalmente en el cómo se debe hacer salud mental en las instituciones sanitarias chilenas. El objetivo fundamental es generar eficiencia y eficacia en el gasto público en salud mental, sin descuidar la calidad asistencial en este ámbito. La gestión se vuelve un mecanismo de control racional de los recursos sanitarios, Lazcano nos dice a propósito que:

en el contexto de una atención sanitaria moderna en países desarrollados, no es posible obviar la necesidad de eficiencia de las organizaciones sanitarias, y por ello de la introducción de criterios de gestión y calidad asistencial en la práctica cotidiana, con un objetivo común: prestar la mejor atención posible, y obtener los mejores resultados con los recursos disponibles. (Chicharro Lezcano, 2012: 726).

El modelo de gestión en salud mental se implanta como un bastión del PNSM, toda vez que se preocupa por los aspectos económicos que se despliegan en procesos asistenciales de los dispositivos de salud mental. Mediante la obtención de indicadores de la estructura de la salud (disponibilidad, accesibilidad), indicadores de procesos de la salud pública (uso, productividad, utilización) y los resultados de estos (eficacia, eficiencia), se busca maximizar los recursos públicos, permitiendo administrar de mejor forma la intervención en salud mental.

Foucault ya nos habia advertido sobre este tipo de lógicas gubernamentales. Este decía que:

en relación a estos fenómenos, la Biopolítica va a introducir [...] mecanismos mucho más sutiles, económicamente mucho más racionales que la asistencia a granel [...]. Vamos a ver mecanismos más sutiles, más racionales, de seguros, de ahorro individual y colectivo, de seguridad, etcétera. (Foucault, 2008:221).

Las estrategias de la salud mental propuestas en el PNSM y el nuevo modelo de gestión perfeccionan las nuevas formas de control, seguridad y vigilancia, optimizando, por un lado, la gestión sanitaria y, por otro, el desarrollo de una seguridad ciudadana (en contra del loco enfermo). En consecuencia, el problema 
biopolítico acá no sería en tanto una articulación conflictiva entre psiquiatría y política, sino más bien la salud como un objeto de la gestión, operando a través de una razón gubernamental, un gobierno de la locura (Foucault, 2007). Esta idea implícita que logra develar el análisis biopolítico no es más que una forma de conocimiento que da cuenta de la mentalidad con que se gobierna a las personas y cómo es posible tal ejercicio, a través de los Estados y las políticas sociales dirigidas a los individuos y la población. Al respecto, Carrasco asegura que: "el poder produce lo pensable y practicable", a partir de lo que Foucault Ilama "las manifestaciones de la verdad". En los estados modernos estas surgen de un ejercicio de poder sobre un objeto que se busca controlar: la población; se necesita conocerla de forma precisa, a fin de gobernarla por reglas racionales, con lo cual el sujeto moderno es constituido como un "objeto de conocimiento", y así es también "convertido en un objeto de control" (Carrasco, 2014: 132). En consecuencia, la racionalidad del Estado se funda en el conocimiento verdadero de lo que se quiere gobernar, en este caso, el gobierno de la locura.

Por ende, la edificación de la psiquiatría y salud mental comunitaria no es más que el triunfo de una estrategia de intervención social que implica un cierto control, más allá de la institucionalidad psiquiátrica. Sobre todo, significa un control territorial de la población, a través de un razonamiento gubernamental, en donde la desinstitucionalización psiquiátrica coincide con la dispersión de las tecnologías de subjetivación y en donde el paciente psiquiátrico es el "locus" de aquellas tecnologías de poder. Sin embargo, para el MINSAL, el objetivo de la psiquiatría comunitaria es:

Contribuir a que las personas, las familias y las comunidades alcancen y mantengan la mayor capacidad posible para interactuar entre sí y con el medio ambiente, de modo de promover el bienestar subjetivo, el desarrollo y uso óptimo de sus potencialidades psicológicas, cognitivas, afectivas y relacionales, el logro de sus metas individuales y colectivas, en concordancia con la justicia y el bien común. (MINSAL, 2000: 3).

En correspondencia con lo dicho anteriormente, la salud mental se constituirá no solo como un resultado de fenómenos biológicos, sino más bien como la configuración de múltiples factores psicosociales que interactúan en la comunidad. Sin embargo, y 
De Prácticas y discursos

Universidad Nacional del Nordeste

Centro de Estudios Sociales

Año 8, Número 11, 2019, Marzo

ISSN 2250-6942

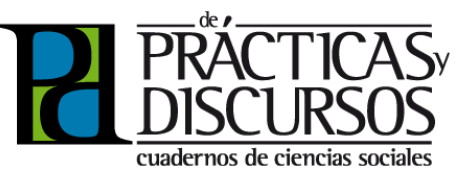

en clave biopolítica, la comunidad se establecerá como un nuevo agente de control poblacional, ahora fuera de las paredes del manicomio, a través de disposiciones tecnológicas que van desde la fármacovigilancia, pasando por la medicalización, hasta la promoción y prevención de la salud mental como formas de poder "hacer vivir". Carrasco (2018) dirá al respecto, "los profesionales encargados de la intervención deben vigilar las trayectorias de los usuarios: si asisten o no al colegio, al trabajo, si se reúnen o no con amigos, si participan o no en organizaciones, etc. Luego deben hacer circular la información" (Carrasco, 2018: 175), y en definitiva la comunidad se transforma en el nuevo panóptico gubernamental. En definitiva, cabe advertir, que las intervenciones que se generarán en la comunidad, por parte de los equipos de salud mental, apuntarán a estrategias de prevención y promoción en sectores marginados o vulnerables (social y biológicamente), con el fin de adherir a estos a una sociedad funcional de competencia. Definitivamente, las disciplinas derivadas de este proceso se constituyen como un punto de contacto entre las tecnologías de control, propias de una lógica neoliberal de gobernanza, y las técnicas del yo.

\section{GESTIÓN DEL SUFRIMIENTO PSÍQUICO}

Para hablar del sufrimiento psíquico desde una perspectiva gubernamental, es necesario, inherentemente, vincularse a la noción de biopolítica como una tecnología de intervención sobre las poblaciones (y por ende en los individuos). En ese sentido, entenderemos a la biopolítica como tecnología derivada de un conjunto de procedimientos que tiene como fin moldear las conductas de las poblaciones e individuos, sostenidas por un conocimiento científico, especialmente estadístico. En otras palabras, la biopolítica es una tecnología gubernamental con el objeto de administrar la vida de las poblaciones, especialmente la vida de los ciudadanos, es decir, la conservación de la vida biológica y su salud. La biopolítica tendrá como objetivo último instalar, para cada riesgo o peligro, ciertos mecanismos de seguridad que tendrian cierta semejanza con los dispositivos disciplinarios, pero que son mucho más sutiles en su dominación.

En este contexto, podríamos decir que el modelo médico del sufrimiento psíquico (Lobo y Huertas, 2018) se expande ilimitadamente a través de una permanente medicalización de la vida cotidiana por medio de politicas gubernamentales que se dirigen 
a las poblaciones, tanto vulnerables en temas relacionados con la salud mental como también a quienes no. De ese modo, la gestión en salud mental, desde el enfoque gubernamental, revela su ajuste con los parámetros de la oferta y la demanda individualizada de los "productos de la psiquiatría", es decir, los psicofármacos, las psicoterapias, las psicoeducaciones, las tecnologías diagnósticas, tratamientos estandarizados, etc.

Esta "gubernamentalización" de la vida de los locos y especialmente del sufrimiento psíquico devela un entramado complejo y oscuro de las relaciones de poder que oculta la salud mental en su naturaleza y que se establecen como verdad. La salud mental y la psiquiatría comunitaria como regímenes de verdad caracterizan al buen gobierno $y$, por tanto, sus formas de operar e intervenir la sociedad (poblaciones e individuos). De algún modo, la psiquiatría, y posteriormente la salud mental, se han hecho cargo de la administración de los cuerpos y emociones en torno al sufrimiento psiquico, desarrollando tecnologias de subjetivación que hacen posible la obediencia ciudadana en torno a estas temáticas. La razón gubernamental de la locura tiene como caracteristica principal la administración del cuerpo y las emociones de los ciudadanos, a partir de una serie de herramientas y procedimientos que administran la libertad de los individuos y su sufrimiento psíquico. Una traducción de lo anterior se podría ejemplificar en el malestar psíquico que pudiera tener un individuo, llámese psicosis, depresión, etc. Estas serán medicalizadas de por vida por el modelo hegemónico psiquiátrico, a través de los dispositivos de seguridad que el gobierno de la locura ha introducido para su control y administración, colonizando el sufrimiento psíquico.

En consecuencia, la psiquiatrización del sufrimiento y su medicalización responden a un engranaje gubernamental que se expresa a través de dispositivos de seguridad que han sido instalados paulatinamente en la retina social. Parafraseando a Foucault, el éxito de ello se debe principalmente a cómo se ha configurado el deseo humano (Foucault, 2008). En ese sentido, el deseo se vuelve una especie de principio de vida de la población, es decir, de cada individuo, el cual en su conjunto provoca el movimiento de la población. Ello tiene como consecuencia que la acción de gobierno consista en hacer concordar el deseo de los individuos con un interés general. En otras palabras, lo que se deja ver en esta operación es la búsqueda direccionada que el gobierno tiene para la creación de un interés para con el individuo. Es de esta 
De Prácticas y discursos

Universidad Nacional del Nordeste

Centro de Estudios Sociales

Año 8, Número 11, 2019, Marzo

ISSN 2250-6942

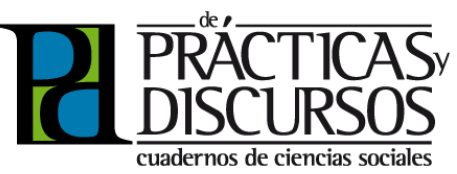

manera como la lógica (neo)liberal penetra la sociedad, subjetivando el sufrimiento psíquico de los individuos y de la población de manera simultánea. Esto se visualiza perfectamente en cómo hoy existe un elevado requerimiento por tener una buena salud mental como característica fundamental del buen ciudadano. Un ejemplo de ello son los altos índices de depresión que escalan exponencialmente en los países desarrollados, además de la proliferación de nuevas enfermedades psiquiátricas que exponen las formas de vida en tiempos globalizados y que son controlados por el consumo (voluntario e involuntario) de psicofármacos, especialmente antidepresivos y ansiolíticos (Gøtszche, 2016).

El moldeamiento de la libertad de conciencia por parte de un gobierno de la locura coincide con esta libertad del poder hacer en tiempos neoliberales. La lógica de libertad ilimitada que tiene el individuo contemporáneo no tiene coacción en principio, sin embargo, genera coacciones, es decir, por ejemplo, la depresión u otras formas del sufrimiento psíquico son realmente la expresión máxima de una crisis profunda de la libertad humana, debiendo crear mecanismos que obliguen a tratar el sufrimiento psíquico o enfermedades mentales. De algún modo, el gobierno de la locura viene a hacer eso, pretende la seguridad de un sistema frente al individuo loco y, por otro lado, mantener el control de las posibles poblaciones vulnerables a desequilibrios neuronales. Como dice Byung Chul Han, "el sujeto [...] que se pretende libre, en realidad es un esclavo" (2015: 12). El ejemplo sobre la explotación de sí mismo queda reflejado en cómo la libertad emerge en el deseo de superación, del éxito, etc., transformándose en la nueva forma de dominación social que absolutiza la vida cotidiana del individuo y, en este caso, la "psiquiatriza". En ese sentido, la salud aparece como un ideal de vida que el neoliberalismo explota de manera eficiente e inteligente. Por ello, el control de las emociones se constituye como una expresión de la perfección de este modo de gobierno, ya que en ellas podemos encontrar el núcleo de dominación del deseo que reside al interior de los individuos. En otras palabras, estar o no estar depresivo son en si mismos moldeamientos de conductas que son explotadas de acuerdo a estrategias diseñadas por una razón gubernamental y neoliberal de la locura, con el fin de mantener el control y la seguridad ciudadana.

En definitiva, cuando hablamos de salud mental en la actualidad, especialmente en Chile, hablamos de una nueva razón guberna- 
mental que manipula intereses (Foucault, 2007) que se despliegan a través de dispositivos de seguridad que emergieron desde la reforma de salud mental en los años noventa hasta el PNSM y el modelo de gestión de los últimos dos años. Su articulación con el sufrimiento psíquico se traduce en un control constante de la vida cotidiana, tanto del loco como de aquello que posiblemente pueda estarlo. Y es en ese punto donde neoliberalismo y salud mental emergen de manera conjunta, pero no transparente, ya que su relación está intimamente ligada a un imperativo del rendimiento (Han: 2016:29). Por un lado, un capitalismo del consumo capitaliza las emociones, adquiriendo relevancia como un recurso de productividad, pero que, por otro lado, se convierte en una afección peligrosa que debe ser constantemente medicalizada y asegurada por dispositivos de seguridad.

Finalmente, la diferencia entre los mecanismos disciplinarios de la locura, como fueron las instituciones totales y el encierro, se distinguirán de los mecanismos de poder biopolítico. La salud mental comunitaria se constituirá como un nuevo agente de control poblacional, ahora fuera de las paredes del psiquiátrico, como un dispositivo de seguridad de la población, a través de tecnologías que van desde lo terapéutico hasta lo psicofarmacológico. En otras palabras, la salud mental hoy no es más que una posinstitución total, o dispositivos ambientales (Rose, 2017), también denominada como extitución (Tirado y Domenech, 2001) o como Pérez (2012: 20) dice: "un manicomio distribuido". Definitivamente, la psiquiatría comunitaria además de constituirse en un entramado de vigilancia y gestión del sufrimiento, se desplaza de sus orígenes hacia un lugar en donde el sujeto loco, producto de sus derechos deja de estar encerrado en un manicomio y "es liberado" en una comunidad, pero bajo un panóptico poblacional. Ello quiere decir, según esta lógica, que indistintamente que existan locos, depresivos, esquizofrénicos o bipolares, lo más importante es prevenir y medicalizar cualquier desajuste neuronal que pueda ser detectado.

\section{A MODO DE CONCLUSIÓN}

Al comenzar este artículo, dijimos que las enfermedades psiquiátricas se han establecido como una de las patologías más importantes en el mundo. La prevalencia de ellas en Chile y en gran parte del mundo se ha convertido en una preocupación 
De Prácticas y discursos

Universidad Nacional del Nordeste

Centro de Estudios Sociales

Año 8, Número 11, 2019, Marzo

ISSN 2250-6942

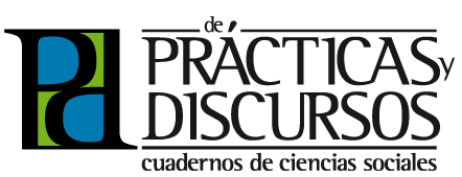

central en los gobiernos e instituciones de salud, lo que ha permitido instalar global y regionalmente, regulaciones importantes sobre la salud mental. Las enfermedades del ánimo, tales como las depresiones, por ejemplo, han sido factores determinantes que han debilitado la fuerza de trabajo, implicando un alto costo económico a los Estados y los sistemas de seguros de salud. Las intervenciones terapéuticas y psicofarmacológicas, como formas de intervención que han desarrollado los gobiernos, tanto en escuelas, organismos de salud como también otro tipo de instituciones, se fundan como estrategias públicas para evitar la proliferación de las enfermedades mentales y abordar de manera oportuna las poblaciones más vulnerables. De ese modo, han emergido de manera sistemática programas relacionados con la prevención y promoción de la salud mental, instalándose como políticas de Estado aplicadas a las poblaciones, que según las prevalencias, son más propensas que otras a tener este tipo de trastornos (estudiantes, trabajadores, sectores pobres, etc.) En otras palabras, la prevención y promoción de la salud mental se cristianizan en estrategias sanitarias (o tecnología biopolítica) que apuntan a tener el control de la salud (y no de la enfermedad) de las personas.

De ese modo, ha emergido todo un engranaje que administra de manera eficiente y eficaz el sufrimiento psíquico, todo ello vinculado a dispositivos de seguridad que mantienen el orden y el control social de la locura. Estos dispositivos se entienden como los que hacen posible los cambios políticos y administrativos del gobierno de la locura. Y como "extituciones" o "manicomios distribuidos", funcionan como mecanismos que permiten la circulación tanto de tecnologías de control como el desplazamiento del propio sujeto, asegurando las trayectorias de los locos en medio de una vigilancia permanentemente. Mientras que, por otro lado, se consolida una mentalidad gubernamental acerca de la locura con una razón que conduce los intereses a través de estos dispositivos, capitalizando las emociones y el sufrimiento psíquico de las personas.

Cuando hablamos de biopolítica, lo que estaría en juego no es necesariamente una política de la vida, sino también prácticas sociales que se ejercen sobre individuos y las poblaciones. En consecuencia, la vida no será un objeto de tematización de las ciencias biológicas, sino que es un espacio ideológico para garantizar la gestión y el control de las poblaciones en las socieda- 
des modernas. La salud mental y la psiquiatría comunitaria pueden leerse en esta clave, permitiendo revelar ciertos discursos -de los que Foucault ya era conciente en sus primeras obras-, pero que hoy en tiempos de economías globales se han convertido en una forma de vivir. En ese sentido que, más que una política sobre la vida, la salud mental comunitaria se establecerá como un modo de gestión y administración de la vida, además de individualizar el sufrimiento psíquico, convirtiéndose en un poder gubernamental que opera a través de políticas de gestión y directrices normativas.

Por último, la salud mental chilena, que camina hacia una senda legislativa, tal como en otros paises latinoamericanos a ocurrido, busca regular sistemáticamente todos los mecanismos relacionados con el tratamiento e intervención en este campo. Lo que se indaga de fondo es poder regularizar las prácticas psiquiátricas que permitirán mejorar los dispositivos de seguridad y como fin último asegurar más años de vida productiva y saludable de la población, evitando en la medida de lo posible, a través de mecanismos de control, la morbilidad y comorbilidad de las enfermedades mentales. Para ello, consolidar medios de gestión en salud mental, desde una perspectiva economicista, permitiria optimizar un gobierno de la salud mental. Si bien los actores del cambio paradigmático de salud mental chileno no han buscado intencionalmente una forma liberal de gestión, no han cuestionado dichas consecuencias, consolidando un modelo acorde a las prácticas neoliberales.

BIBLIOGRAFIA

BASAGLIA, F. (1972). La Institución Negada, Informe de un hospital psiquiátrico. España: Barral Editores.

(2008). La Condena de ser loco y pobre: Alternativas al manicomio. Buenos Aires: Topia Editorial.

BLEULER, E. (1924). Texbook of Psychiatry. New York: Macmillan.

CARRASCO, J. (2014). Salud Mental y Psiquiatría Comunitaria en Chile: el proceso de configuración de un objetivo de gobierno. En Karmy, R. y Tuillang, Y. (comps.) Biopolítica, Gobierno y Salud Pública. Miradas para un diagnóstico diferencial. Escuela 
de Salud Pública, Universidad de Chile (pp. 149). Santiago: Ocho Libros Editores.

CARRASCO, J. Y LEAL, A. (2018). La reforma psiquiátrica brasileña y chilena y la gestión por la libertad. Una historia de los modos de gobierno en las prácticas vinculadas a la salud mental. En Karmy, R. y Follegati, L. (comps.) Estudios en Gubernamentalidad. Ensayos sobre el poder, vida y neoliberalismo (pp.161-194). Santiago: Communes.

COOPER, DAVID (1976). Psiquiatría y Antipsiquiatría. Buenos Aires: Locus Hipocampus Editorial.

CHICHARRO LEZCANO, F. (2012). La Gestión de Calidad. En Desviat, M. y Moreno, A. (comps.) Acciones de la Salud Mental en la Comunidad (p. 726). Madrid: Asociación Española de Neuropsiquiatría, Estudios 47.

DESVIAT, M. Y MORENO, A. (CONT.) (2012) Acciones de Salud Mental en la Comunidad. Madrid: Asociación Española de Neuropsiquiatría, Estudios 47.

DELEUZE, G. (1996). Conversaciones. Valencia: Pre-Textos.

DESVIAT, M. Y MORENO, A. (EDS.) Acciones de Salud Mental en la Comunidad. Madrid: AEN.

FOUCAULT, M. (1996). La Vida de los Hombres Infames. Argentina: Editorial Altamira.

(2002). La Arqueología del Saber. Buenos Aires: Siglo XXI Editores.

(2007). El Nacimiento de la Biopolítica. Buenos Aires: Fondo Cultura Económica.

(2008). Defender la Sociedad. Buenos Aires: Fondo Cultura Económica.

GABAY, P. (1999). Rehabilitación y reinserción social en salud mental, perspectivas internacionales. Vertex Revista Argentina de psiquiatría, (X)36, julio-agosto. 
GOLDBERG, D. Y HUXLEY, P. (1990). Enfermedad Mental en la Comunidad. Madrid: Nieva.

GOFFMAN, E. (1998). Internados: Ensayos sobre la situación social de los enfermos mentales. Buenos Aires: Amorrortu Editores.

GØTSZCHE, P. (2016). Psicofármacos que matan y denegación organizada. Barcelona: los Libros del Lince.

HAN, BYUNG-CHUL (2015). Psicopolítica. Barcelona: Herder.

(2016). La Sociedad del Cansancio. Barcelona: Herder.

Kraepelin, E. (1913). Dementia Praecox. En Kraepelin, E. Psychiatrica, 8a ed. Florida: Krieger.

LAING, R. (1975). El yo dividido: Un estudio sobre la Salud y Enfermedad. México: Fondo Cultura Económica.

MINOLLETTI, A.; SEPÚLVEDA, R.; NARVÁEZ, P. Y CAPRILE, A. (2010). Chile: lecciones aprendidas en la implementación de un modelo comunitario de atención en salud mental. En Rodríguez, J. (comp.) Salud Mental en la Comunidad (pp. 339). Buenos Aires: OPS.

ORGANIZACIÓN MUNDIAL DE LA SALUD (2013). Plan de Acción sobre Salud Mental 2013-2020: Ginebra: Autor.

ORTIZ LOBO, A. Y HUERTAS, R. (COMP.) (2018). Críticas y alternativas en psiquiatria. Madrid: Cataratas Ediciones.

PEMJEAN, A. (2006). In Memmoriam. Cuadernos de Psiquiatría Comunitaria, (6)1. España.

PÉREZ SOTO, C. (2012). Una nueva Antipsiquiatría, crítica y conocimiento de las técnicas de control psiquiátrico. Santiago: LOM editores.

MINSAL, REPÚBLICA DE CHILE, (1993). Estrategia de Salud Mental. Santiago: Gobierno de Chile.

(2000). Política y Plan Nacional de Salud Mental. Santiago: Gobierno de Chile. 
(2014). Sistema de Salud Mental de Chile: Segundo Informe. Santiago: Gobierno de Chile.

(2017). Política y Plan Nacional de Salud Mental. Santiago: Gobierno de Chile.

(2018). Modelo de Gestión: Red Temática de Salud Mental en la red general de salud. Santiago: Gobierno de Chile.

MINSAL, REPÚBLICA DE CHILE, DIRECCIÓN DE RECTORÍA Y REGULACIÓN SANITARIA (2000). Norma Técnica Hogares Protegidos. Santiago: Gobierno de Chile.

PINEL, P. (1988). Tratado médico filosófico de la enajenación mental o la manía. España: Ediciones Nieva.

READ, J. Y GEEKIE, J. (2012). El Sentido de la Locura. BarceIona: Herder Editorial.

ROSE, N. (2007). Terapia y poder: techné y ethos. Cuadernos crítica de la cultura, Archipiélago, 76, 101-124.

TIRADO, F. Y DOMÉNECH, M. (2001). Extituciones: del poder y sus anatomías. Políticas y Sociedad, 36, 191-204.

SZASZ, T. (1970). Ideología y Enfermedad Mental. Argentina: Amorrortu Editores. 